\title{
Salidroside inhibits endothelial-mesenchymal transition via the KLF4/eNOS signaling pathway
}

\author{
YONGPAN HUANG $^{1 *}$, XIAODONG HAN ${ }^{2,3^{*}}$, JIAYU TANG $^{4 *}$, XIAN LONG ${ }^{1}$ and XIAOYE WANG \\ ${ }^{1}$ Department of Clinic, Medicine School, Changsha Social Work College, Changsha, Hunan 410004; \\ ${ }^{2}$ Department of Anesthesia, Medical College, Yan'an University, Yan'an, Shanxi 716000; \\ ${ }^{3}$ Department of Anesthesia, Xi'an International Medical Center Hospital, Xi'an, Shanxi 710100; \\ ${ }^{4}$ Department of Neurology, Brain Hospital of Hunan Province, Changsha, Hunan 410007, P.R. China
}

Received February 5, 2021; Accepted May 28, 2021

DOI: $10.3892 / \mathrm{mmr} .2021 .12324$

\begin{abstract}
Homocysteine (Hcy) was discovered to be an independent risk factor for the development of atherosclerosis (AS). Moreover, endothelial-mesenchymal transition (EndMT) was found to be one of main mechanisms contributing to the pathogenesis of AS. Salidroside (SAL) has diverse pharmacological activities, including anti-inflammatory, anti-cancer, anti-oxidative and anti-fibrosis properties. However, whether SAL serves a beneficial role in Hcy-induced EndMT remains unknown. The present study aimed to investigate whether SAL exerted its effects on Hcy-induced EndMT via the Kruppel-like factor 4 (KLF4)/endothelial nitric oxide (NO) synthase (eNOS) signaling pathway. HUVECs were pretreated with high and low doses (10 or $50 \mu \mathrm{mol} / \mathrm{l})$ of SAL for $2 \mathrm{~h}$, followed by $1 \mathrm{mmol} / 1 \mathrm{Hcy}$ for $48 \mathrm{~h}$ to induce EndMT. Western blotting was used to analyze the protein expression levels of the endothelial marker, VE-cadherin, the mesenchymal cell marker, $\alpha$-smooth muscle actin (SMA), and the nuclear transcription factors, KLF4 and eNOS. Wound healing assays were used to determine the cell migratory ability, and the levels of NO in the cell culture supernatants were measured using a nitrate reductase assay. Cellular immunofluorescence was used to analyze the expression and localization of KLF4. Small interfering (si)RNA targeting KLF4 (siKLF4) was used to knock down KLF4 expression in HUVECs. The results of the present study revealed that treatment with SAL upregulated the expression levels of VE-cadherin, downregulated the expression levels of $\alpha$-SMA, reduced cell migration and activated the eNOS/NO signaling axis, as well
\end{abstract}

Correspondence to: Professor Xiaoye Wang, Department of Neurology, Brain Hospital of Hunan Province, 427 Furong Middle Road, Changsha, Hunan 410007, P.R. China

E-mail: hnnkyy_xiaoyewang@163.com

${ }^{*}$ Contributed equally

Key words: salidroside, endothelial-mesenchymal transition, Kruppel-like factor 4, endothelial nitric oxide synthase as downregulated KLF4 expression and translocation to the nucleus. Compared with the SAL + siKLF4 co-administration group, no significant differences were observed in the expression levels of the phenotypic markers in the SAL or siKLF4 groups. In conclusion, the findings of the present study revealed that SAL may inhibit Hcy-induced EndMT via regulation of the KLF4/eNOS signaling pathway.

\section{Introduction}

Injury to vascular endothelial cells was found to alter the biological functions of these cells via the activation of endothelial-mesenchymal transition (EndMT) (1). EndMT is an intricate cellular differentiation process, whereby endothelial cells lose their original endothelial properties and, to varying extents, acquire mesenchymal features (2), which has been discovered to be an important pathological process in the progression and evolution of atherosclerosis (AS) (3). The decreased activity of endothelial nitric oxide (NO) synthase (eNOS) and decreased NO bioavailability have been established as significant features of endothelial function injury (4). eNOS is a key enzyme required by endothelial cells to synthesize $\mathrm{NO}$ and the expression level of eNOS has been shown to serve an important role in EndMT-mediated AS-related diseases (5). Therefore, the eNOS/NO signaling axis has been suggested to be involved in the regulation of vascular function during EndMT.

Transcription factors (TFs) regulate a large number of cellular activities by binding to specific target DNA sequences. Kruppel-like factors (KLFs) are zinc finger TFs, which serve crucial roles in regulating important biological processes, such as cell proliferation, the differential expression of phenotypes and cytoskeletal remodeling $(6,7)$. Therefore, the present study hypothesized that the KLF4/eNOS signaling pathway may be involved in the EndMT process.

Salidroside (SAL) is the main active ingredient of Rhodiola rosea, which belongs to the Crassulaceae family. Previous studies have reported that SAL could inhibit oxidative stress, inflammation and endoplasmic reticulum stress in vascular endothelial cells, as well as delay endothelial cell senescence, thereby exerting a strong cardioprotective pharmacological effect (8-13). A number of studies have also revealed that SAL 
treatment upregulated the expression levels of the homocysteine (Hcy)-induced endothelial cell antioxidant signaling protein, nuclear factor, erythroid 2 like 2 (Nrf2), and improved endothelial cell oxidative stress injury, suggesting that SAL may have the potential to protect endothelial cells (12-14). Therefore, the present study aimed to investigate whether the effect of SAL on Hcy-induced EndMT occurred via the KLF4/eNOS signaling pathway, which may provide experimental evidence of the vascular pharmacological activity of SAL.

\section{Materials and methods}

Reagents. SAL was purchased from Shanghai Aladdin Biochemical Technology Co., Ltd. (purity $\geq 98 \%$; cat. no. B19208). Endothelial cell culture medium (cat. no. 26219) and cell cryopreservation solution (cat. no. 19671) were purchased from ScienCell Research Laboratories, Inc. VE-cadherin (1:1,000; cat. no. 2500), $\alpha$-smooth muscle actin $(1: 1,000$; $\alpha$-SMA; cat. no. 19245), KLF4 (1:1,000; cat. no. 4038) and eNOS (1:1,000; cat. no. 9572) primary antibodies were purchased from Cell Signaling Technology, Inc. The GAPDH primary antibody (cat. no. MB001) was purchased from Bioworld Technology, Inc. The BCA protein quantitative kit (cat. no. P0010) was purchased from Beyotime Institute of Biotechnology, while the ECL detection kit (cat. no. 1829501) was purchased from MilliporeSigma and small interfering RNA (siRNA/si) was purchased from Shanghai Gema Gene Technology Co., Ltd.

Cell lines, culture and experimental groupings. HUVECs (cat. no. 19195) were purchased from ScienCell Research Laboratories, Inc. HUVECs were cultured in DMEM (cat. no. 56499C; Sigma-Aldrich; Merck KGaA) supplemented with 5\% FBS (cat. no. F8687; Sigma-Aldrich; Merck KGaA), $1 \%$ penicillin-streptomycin and $1 \% \mathrm{EGF}$, and maintained at a constant temperature of $37^{\circ} \mathrm{C}$ with $5 \% \mathrm{CO}_{2}$ in an incubator until the cell confluence reached $80-90 \%$.

For the first experiment (experiment 1), cells were divided into the following groups: i) Control group; ii) Hcy (model) group [1 mmol/1 Hcy (Sigma-Aldrich; Merck KGaA)]; iii) SAL low-dose group ( $1 \mathrm{mmol} / 1 \mathrm{Hcy}+10 \mu \mathrm{mol} / 1 \mathrm{SAL})$; and iv) SAL high-dose group ( $1 \mathrm{mmol} / 1 \mathrm{Hcy}+50 \mu \mathrm{mol} / 1 \mathrm{SAL})$. Cells were pretreated with SAL for $2 \mathrm{~h}$, then treated with Hcy for $48 \mathrm{~h}$, both at $37^{\circ} \mathrm{C}$ to induce EndMT.

For the second experiment (experiment 2), siRNAs were used to knock down the expression of KLF4 in HUVECs, and whether the inhibitory effects of SAL on EndMT occurred via the KLF4/eNOS signaling pathway were investigated. Cells were divided into the following groups: i) Control group; ii) Hcy group (1 mmol/1 Hcy); iii) SAL group ( $1 \mathrm{mmol} / 1 \mathrm{Hcy}+50 \mu \mathrm{mol} / 1 \mathrm{SAL})$; iv) siKLF4 group $[1 \mathrm{mmol} / 1 \mathrm{Hcy}+5 \mu \mathrm{l}$ siKLF4 $(20 \mu \mathrm{M})]$; and iv) siKLF4 + SAL co-administration group $[1 \mathrm{mmol} / 1 \mathrm{Hcy}+5 \mu 1 \mathrm{siKLF} 4$ $(20 \mu \mathrm{M})+50 \mu \mathrm{mol} / 1 \mathrm{SAL}$. To induce EndMT, all groups were preincubated with SAL for $2 \mathrm{~h}$ and then treated with Hcy for $48 \mathrm{~h}$ at $37^{\circ} \mathrm{C}$.

siRNA transfection. Control siRNA (sense, 5'-UUCUCCGAA CGUGUCACGUTT-3' and antisense, 5'-ACGUGACACGUU
CGGAGAATT-3') and siKLF4 (sense, 5'-GGACUUUAU UCUCUCCAAUTT-3' and antisense, 5'-AUUGGAGAGAAU AAAGUCCTT-3') were transfected into HUVECs. Briefly, HUVECs were cultured to $60 \%$ confluence and transfected with $5 \mu \mathrm{l}$ siRNA at $37^{\circ} \mathrm{C}$ using Lipofectamine ${ }^{\circledR} 2000$ (Invitrogen; Thermo Fisher Scientific, Inc.) diluted in serum-free RPMI-1640 medium (Wisent Biotechnology). Following $6 \mathrm{~h}$ of transfection, the cell culture medium was replaced with antibiotic-free endothelial cell culture medium supplemented with 5\% FBS and cultured in normal conditions for $24 \mathrm{~h}$, after which cells were used for subsequent experiments. The transfection efficiency was analyzed using western blotting.

MTT assay. Cells from at least six wells/experimental group were used. Briefly, after a $24 \mathrm{~h}$ exposure to Hcy $(1 \mathrm{mmol} / \mathrm{l})$ at $37^{\circ} \mathrm{C}$, the medium was removed, and the cells were gently washed with PBS. Then, $80 \mu \mathrm{l}$ fresh culture medium and $20 \mu \mathrm{l}$ MTT $(5 \mathrm{mg} / \mathrm{ml})$ were added to the cells. After $4 \mathrm{~h}$ of incubation at $37^{\circ} \mathrm{C}, 200 \mu \mathrm{l} \mathrm{DMSO}$ was added to dissolve the formazan crystals, and the absorbance was measured using a microplate reader (Tecan Group, Ltd.) at a wavelength of $570 \mathrm{~nm}$. Inhibition of cell viability (\%) was calculated with the following formula: [Optical density (OD) $\left.)_{\text {treated group }}-\mathrm{OD}_{\text {ox-LDL group }}\right) /\left(\mathrm{OD}_{\text {control group }}{ }^{-}\right.$ $\left.\left.\mathrm{OD}_{\text {ox-LDL group }}\right)\right] \mathrm{x} 100$.

Lactate dehydrogenase ( $L D H$ ) assay. To evaluate cell injury, $\mathrm{LDH}$ released from the cytosol into the culture medium was measured. After treatment with SAL (10 or $50 \mu \mathrm{mol} / \mathrm{l}$ ) for $1 \mathrm{~h}$, followed by incubation with Hcy $(1 \mathrm{mmol} / \mathrm{l})$ for $24 \mathrm{~h}$ at $37^{\circ} \mathrm{C}$, the medium was collected from each well. Supernatants were obtained by centrifugation at $12,000 \mathrm{x} \mathrm{g}$ at $4^{\circ} \mathrm{C}$ for $10 \mathrm{~min}$. LDH release was determined using an LDH assay kit (Nanjing Jiancheng Bioengineering Institute), according to the manufacturer's protocol. The absorbance was measured at a wavelength of $440 \mathrm{~nm}$ using a microplate reader (SpectraMax 190; Molecular Devices, LLC).

Western blotting. Total protein was extracted from HUVECs using a protein lysate buffer (cat. no. P0013; Beyotime Institute of Biotechnology) supplemented with $1 \% \mathrm{PMSF}$ (cat. no. 36978; Thermo Fisher Scientific, Inc.). Total protein was quantified using a BCA protein assay kit (Abcam) and $40 \mu \mathrm{g}$ protein/lane was separated via 10 or $12 \%$ SDS-PAGE. The separated proteins were subsequently transferred onto PVDF membranes at room temperature and blocked with $10 \%$ non-fat dried milk diluted in TBS-0.1\% Tween-20 (TBST) for $15 \mathrm{~min}$ at room temperature. The membranes were then incubated with the primary antibodies overnight at $4^{\circ} \mathrm{C}$. Following the primary antibody incubation, the membrane was washed with TBST and incubated with a HRP-conjugated secondary (1:10,000; cat. no. BM2020; Wuhan Boster Biological Technology, Ltd.) at room temperature for $2 \mathrm{~h}$. The membranes were washed with 3X TBS-Tween-20 (0.1\%) and total protein was visualized using an ECL reagent (Amersham; Cytiva). Densitometric analysis was performed using ImageJ version 1.43 software (National Institutes of Health).

Detection of changes in KLF4 levels using reverse transcription-quantitative PCR (RT-qPCR). Total RNA was extracted from HUVECs using TRIzol ${ }^{\circledR}$ reagent (Invitrogen; 
Thermo Fisher Scientific, Inc.) and quantified using a NanoDrop ND-2000 system (Thermo Fisher Scientific, Inc.). cDNA was synthesized from the purified RNA (200 ng/sample) using a reverse transcription kit (cat. no. DRR037A; Takara Bio, Inc.), according to the manufacturer's protocol. Subsequently, qPCR was performed using the CFX96 real-time PCR detection system (Bio-Rad Laboratories, Inc.) and Premix Ex Taq kit PCR reagents (cat. no. RR390A; Takara Bio, Inc.) with primers specific for KLF4 and GADPH. The following thermocycling conditions were used for the qPCR: Initial denaturation at $94^{\circ} \mathrm{C}$ for $30 \mathrm{sec}$; followed by 39 cycles of amplification at $94^{\circ} \mathrm{C}$ for $5 \mathrm{sec}$ and $56^{\circ} \mathrm{C}$ for $30 \mathrm{sec}$. The following primer sequences were used for the qPCR: KLF4 forward, 5'-GCATGTGCC CCAAGATTAAG-3' and reverse, 5'-GTGACAGTCCCTGCT GTTCA-3'; and GAPDH forward, 5'-TGTGAACGGATT TGGCCGTA-3' and reverse, 5'-GATGGTGATGGGTTTCCC GT-3'. GAPDH was used as the loading control. Expression levels were quantified using the $2^{-\Delta \Delta C q}$ method (15) and target gene expression was normalized to GAPDH expression.

Wound healing assay. Cells were plated into a 6-well plate and cultured overnight to confluence, then treated according to the experimental groups described as experiment 1. Subsequently, an artificial wound was created by vertically scratching the cell monolayer using a $10-\mu 1$ pipette tip. The non-adherent cells were removed by washing with PBS and cells were incubated for $10 \mathrm{~min}$ at $37^{\circ} \mathrm{C}$ with serum-free medium. Cells were visualized in five randomly selected fields of view at 0 and $12 \mathrm{~h}$ to determine the influence of SAL on cell migration. The following equation was used to calculate the migration using images captured with a fluorescence microscope (Leica DM3000k magnification, x100): Migration $(\%)=[(0 \mathrm{~h}$ average scratch distance- $12 \mathrm{~h}$ average scratch distance) $/ 0 \mathrm{~h}$ average scratch distance] $\mathrm{x} 100$.

Determination of $\mathrm{NO}$ content using a nitrate reductase assay. Following centrifugation at $3,000 \mathrm{x}$ for $10 \mathrm{~min}$ at $4^{\circ} \mathrm{C}$, the cell culture supernatant was collected and analyzed using a commercially available kit (cat. no. S0021S; Beyotime Institute of Biotechnology), according to the manufacturer's protocol.

Immunofluorescence assay. Immunofluorescence was used to analyze the expression levels of KLF4. Briefly, the cells were treated according to the groups described as experiment 2. The cells were washed with PBS, fixed with $4 \%$ paraformaldehyde for $15 \mathrm{~min}$ at room temperature, washed with PBS and permeabilized with Triton X-100 for $10 \mathrm{~min}$. Then, cells were washed with PBS and blocked with $4 \%$ goat serum (Abcam) at room temperature for $1 \mathrm{~h}$. The cells were then incubated with a primary anti-KLF4 antibody (1:1,000; cat. no. 12173S; Cell Signaling Technology, Inc.) overnight at $4^{\circ} \mathrm{C}$. Following the primary antibody incubation, the cells were washed with PBS and incubated with the corresponding fluorescent-conjugated secondary antibody (1:1,000; cat. no. ab150077; Abcam) for $1 \mathrm{~h}$. After washing with PBS, nuclei were stained with DAPI at room temperature for $10 \mathrm{~min}$. Finally, the cells were washed with PBS for $5 \mathrm{~min}$, the fluorescence was quenched, and stained cells were visualized using an inverted fluorescence microscope (magnification, $\mathrm{x} 200$ ) to analyze the expression of KLF4.
Statistical analysis. Statistical analysis was performed using GraphPad Prism-6.0 software (GraphPad Software, Inc.) and all data are presented as the mean \pm SD of at least three experimental repeats. Statistical differences between groups were determined using one-way ANOVA followed by Tukey's post-hoc test. $\mathrm{P}<0.05$ was considered to indicate a statistically significant difference.

\section{Results}

Effect of SAL on the expression levels of Hcy-induced EndMT phenotypic markers. The first series of experiments investigated endothelial cell viability and cell damage. Endothelial cell viability was detected using an MTT assay. Hcy caused a decrease in cell viability in a concentration-dependent manner, especially at $48 \mathrm{~h}$, and the extent of cellular injury at the concentration of $1 \mathrm{mM}$ Hcy was the most severe (Fig. S1A and B). Cell damage was determined by the release of $\mathrm{LDH}$, which increases with necrosis in cultured cells. LDH leakage significantly increased with Hcy exposure, which was in line with the results of cell viability (Fig. S1D). Moreover, SAL improved the cell viability, with a significant decrease of the release of LDH compared with Hcy exposure (Fig. S1C and D).

To further investigate the effects of SAL on the expression levels of Hcy-induced EndMT phenotypic markers, western blotting was performed. As shown in Fig. 1, Hcy significantly downregulated the expression levels of the endothelial marker VE-cadherin and significantly upregulated the expression levels of the mesenchymal marker $\alpha$-SMA in HUVECs. Conversely, compared with the Hcy group, pretreatment with 10 or $50 \mu \mathrm{mol} / 1 \mathrm{SAL}$ upregulated the expression levels of VE-cadherin and downregulated $\alpha$-SMA expression in HUVECs. These results suggested that SAL pretreatment may inhibit Hcy-induced changes in the expression levels of endothelial cell phenotypic markers.

Effect of SAL on Hcy-induced cell migration. As presented in Fig. 2, compared with the control group, the results of the wound healing assay revealed that Hcy significantly increased the cell migration rate $(150.69 \pm 21.97 \%)$, while compared with Hcy group, 10 or $50 \mu \mathrm{mol} / 1 \mathrm{SAL}$ pretreatment decreased the cell migratory rates to $137.79 \pm 11.42$ and $105.58 \pm 10.44 \%$, respectively, suggesting that SAL treatment may inhibit the Hcy-induced cell migratory ability.

SAL regulates the eNOS/NO signaling axis in Hcy-induced EndMT. To investigate the role of eNOS in Hcy-induced EndMT, the expression levels of eNOS were analyzed using western blotting. The results demonstrated that Hcy downregulated the expression levels of eNOS, while SAL pretreatment upregulated the expression levels of eNOS in HUVECs (Fig. 3A and B). To further confirm the role of the eNOS/NO signaling axis in Hcy-induced EndMT, the NO content in the cell culture supernatant was measured (Fig. 3C), and similar trends to the eNOS expression levels were observed. Collectively, it was shown that Hcy downregulated the protein expression level of eNOS and the levels of NO. Moreover, these findings indicated that SAL treatment may upregulate the Hcy-induced downregulated eNOS/NO signaling axis, which 


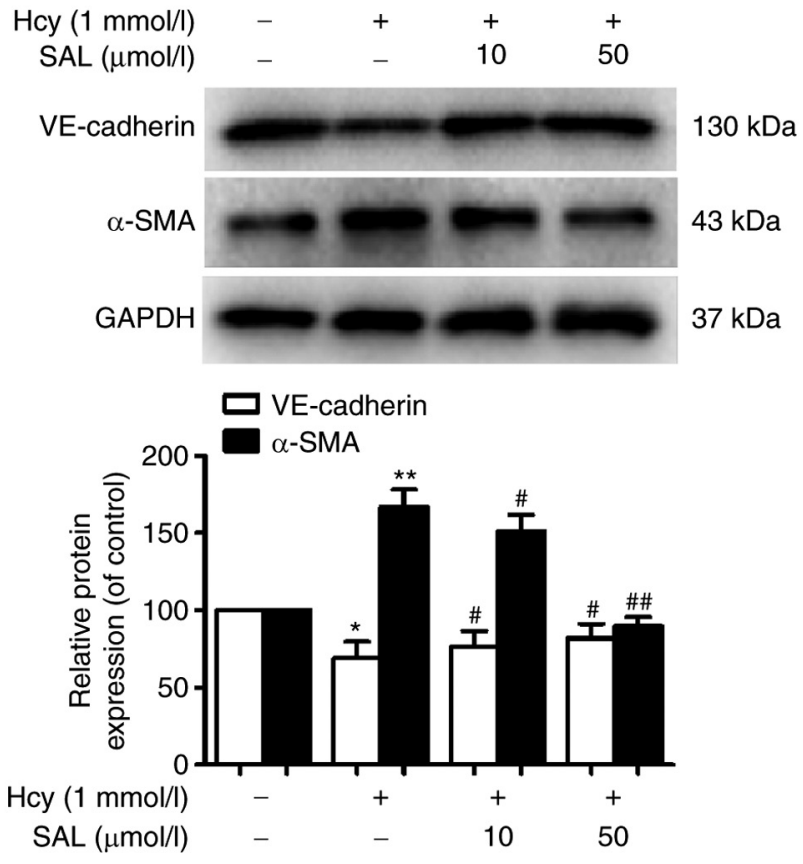

Figure 1. Effects of SAL on the expression levels of Hcy-induced endothelial-mesenchymal transition-related markers. Data are presented as the mean $\pm \mathrm{SD}$ of three independent experiments. ${ }^{*} \mathrm{P}<0.05,{ }^{* *} \mathrm{P}<0.01$ vs. control; ${ }^{\#} \mathrm{P}<0.05,{ }^{\# \#} \mathrm{P}<0.01$ vs. Hcy. Hcy, homocysteine; SAL, salidroside; $\alpha$-SMA, $\alpha$-smooth muscle actin.
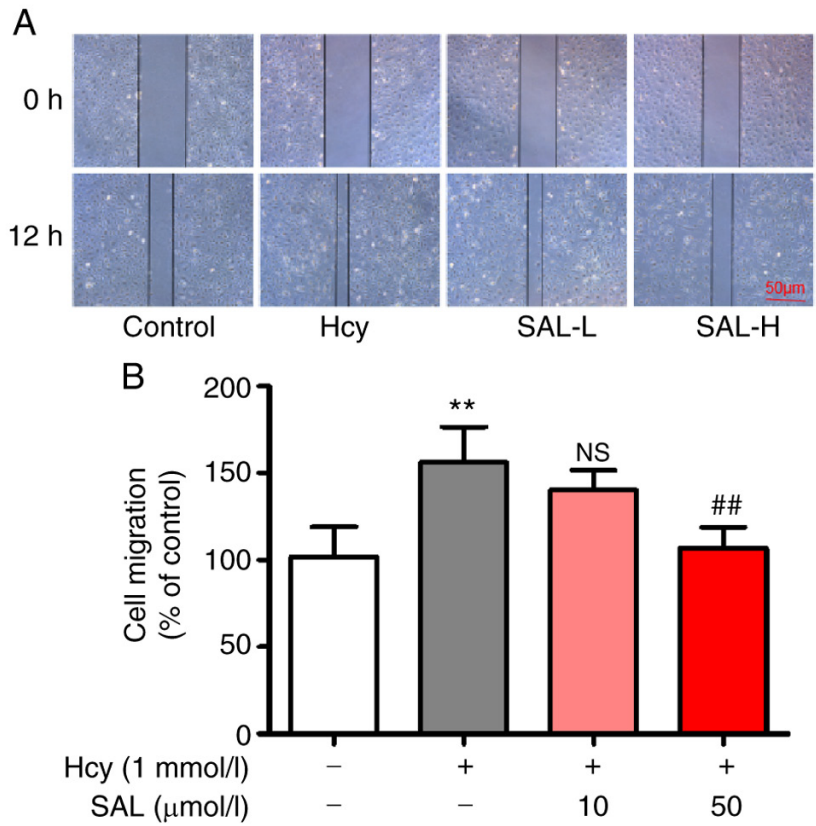

Figure 2. Effect of SAL on the Hcy-induced migration of HUVECs. Scale bar, $50 \mu \mathrm{m}$. (A) Representative images of wound healing assay. (B)Relative migration rate. Data are presented as the mean $\pm \mathrm{SD}$ of three independent experiments. ${ }^{* *} \mathrm{P}<0.01$ vs. control; ${ }^{\# \#} \mathrm{P}<0.01$ vs. Hcy. Hcy, homocysteine; $\mathrm{SAL}$, salidroside; NS, not significant; L, low; H, high concentration.

suggested that SAL may promote the recovery of endothelial dysfunction.

Effect of SAL on the expression levels of KLF4 in HUVECs. Western blotting and RT-qPCR analysis revealed that
A

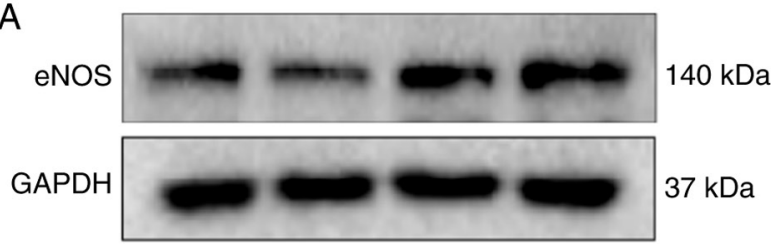

B

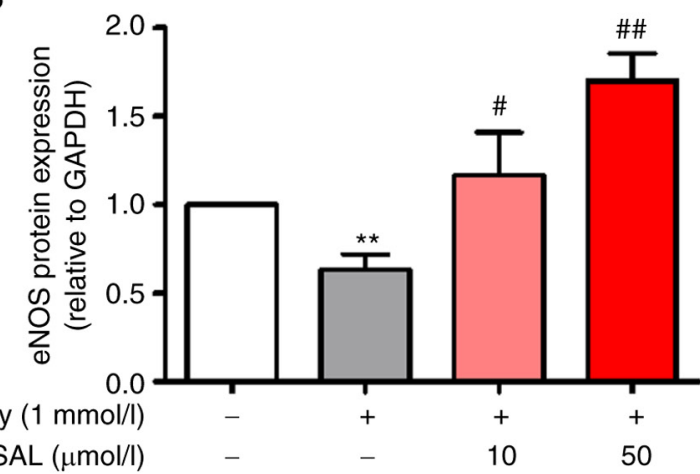

C

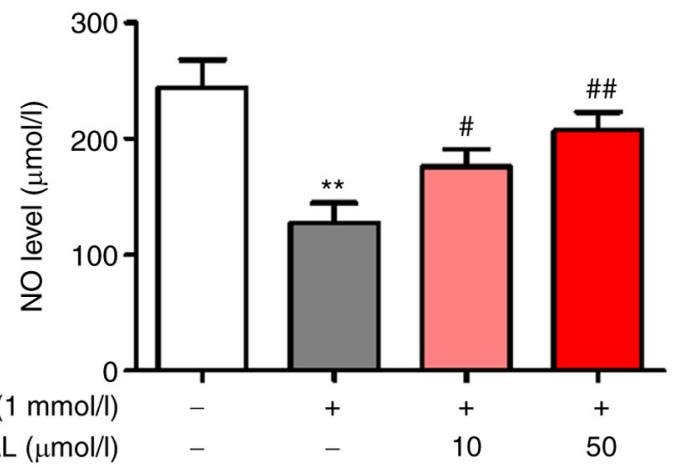

Figure 3. Effect of SAL on eNOS expression in Hcy-induced HUVECs. (A) Expression level of eNOS was analyzed using western blotting, and (B) the results were semi-quantified. (C) NO levels were measured using a nitrate reductase assay. Data are presented as the mean $\pm \mathrm{SD}$ of three independent experiments. ${ }^{* *} \mathrm{P}<0.01$ vs. control; ${ }^{~} \mathrm{P}<0.05,{ }^{\# \#} \mathrm{P}<0.01$ vs. Hcy. Hcy, homocysteine; SAL, salidroside; eNOS, endothelial nitric oxide synthase; NO, nitric oxide.

pretreatment with low and high doses of SAL significantly downregulated the Hcy-induced upregulation of KLF4 expression in HUVECs (Fig. 4A-C). The results of the immunofluorescence analysis further demonstrated that, compared with the control group, Hcy induced the translocation of the nuclear TF KLF4 from the cytoplasm to the nucleus, while $50 \mu \mathrm{mol} / 1 \mathrm{SAL}$ pretreatment decreased the fluorescence intensity of KLF4 and inhibited KLF4 nuclear translocation (Fig. 4D). These results suggested that SAL may effectively downregulate the expression levels of the nuclear TF KLF4 and inhibit the nuclear translocation of KLF4.

Effect of SAL and siKLF4 on the expression levels of Hcy-induced EndMT phenotypic markers, KLF4 and eNOS. To determine whether KLF4 could act as an upstream signaling factor to regulate the expression levels of eNOS and exert a role in the regulation of Hcy-induced EndMT, siKLF4 was used to knock down KLF4 expression. The results revealed that siKLF4 significantly downregulated the expression levels of KLF4, confirming that the transfection 
A
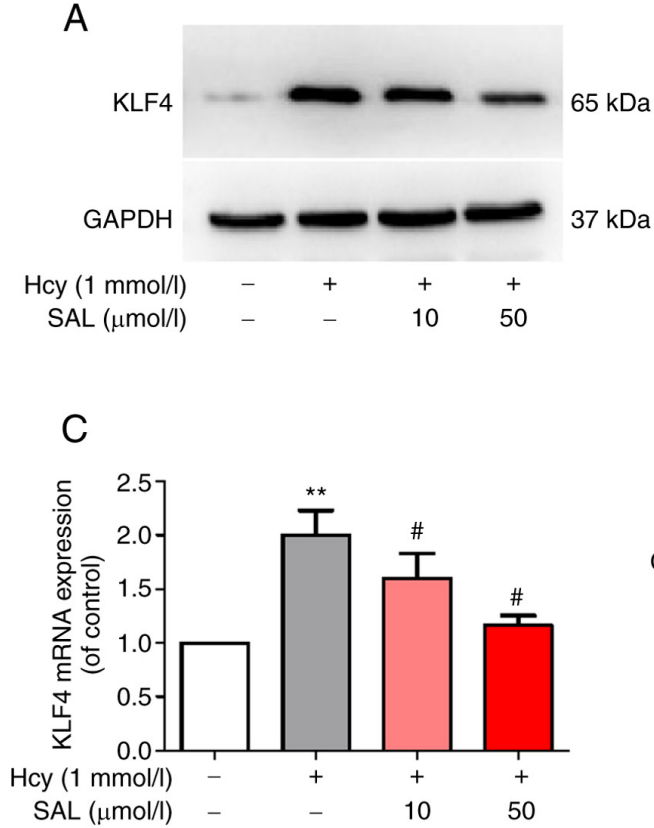

$\mathrm{B}$

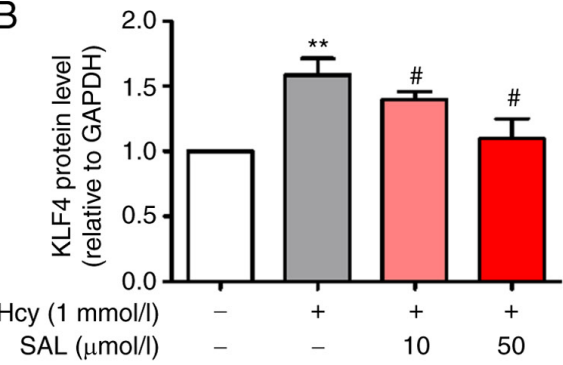

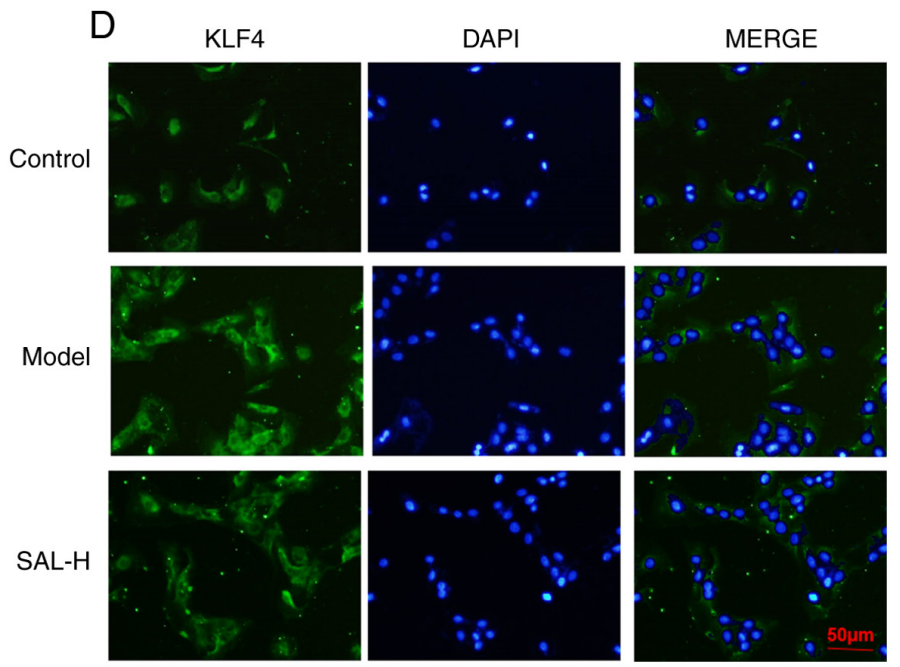

Figure 4. Effect of SAL on the expression levels of KLF4 in Hcy-induced HUVECs. (A) Expression of KLF4 was analyzed using western blotting. (B) Histogram of KLF4 protein expression. (C) Expression of KLF4 was analyzed using reverse transcription-quantitative PCR. (D) Effect of SAL on the Hcy-induced translocation of KLF4 from the cytoplasm to the nucleus. Scale bar, $50 \mu \mathrm{m}$. Data are presented as the mean \pm SD of three independent experiments. ${ }^{* *} \mathrm{P}<0.01$ vs. untreated control; " $\mathrm{P}<0.05$ vs. Hcy. Hcy, homocysteine; SAL, salidroside; KLF4, Kruppel-like factor 4; H, high concentration.

of siKLF4 into HUVECs was successful (Fig. 5A). The results of the western blot analysis demonstrated that Hcy upregulated the expression levels of KLF4 and $\alpha$-SMA in HUVECs, and downregulated the expression levels of eNOS and the endothelial marker, VE-cadherin. Conversely, SAL treatment and KLF4-knockdown inhibited Hcy-induced EndMT by upregulating the expression levels of eNOS and VE-cadherin and downregulating the expression levels of KLF4 and $\alpha-S M A$ (Fig. 5B and C). Compared with the SAL + siKLF4 co-administration group, no significant differences were observed in the expression levels of the phenotypic makers in the SAL or siKLF4 groups. These results suggested that KLF4 may be an important signaling factor involved in the inhibitory mechanism of SAL on Hcy-induced EndMT, and that KLF4 may represent a potential upstream signaling protein to target to regulate eNOS expression. These results indicated that SAL could inhibit Hcy-induced EndMT by downregulating the KLF4/eNOS signaling pathway.

\section{Discussion}

EndMT is the mechanism via which endothelial cells undergo transdifferentiation into a mesenchymal phenotype under the action of internal and external damage factors (16). EndMT can damage the endothelial cell microenvironment by destroying the cell surface and secretory function of endothelial cells, which thereby aggravates endothelial dysfunction (17). Thus, determining key molecules involved in the process of EndMT remains an urgent priority. The findings of the present study demonstrated that Hcy upregulated the expression levels of the mesenchymal cell marker $\alpha$-SMA, KLF4 and the eNOS/NO signaling axis, and downregulated VE-cadherin expression, as well as promoted cell migration and KLF4 translocation to the nucleus. Moreover, the knockdown of KLF4 reversed the aberrant expression of the aforementioned proteins induced by Hcy treatment. SAL treatment could also reverse the Hcy-induced effects. These findings suggested that SAL may provide vascular protection by inhibiting EndMT via the KLF4/eNOS signaling pathway.

Endothelial cell dysfunction is an early factor of AS; therefore, devising innovative drugs that protect vascular endothelial cell function and reverse the biological dysfunction of endothelial cells is of great significance for the prevention and treatment of AS (18). Previous studies have reported that endothelial dysfunction was accompanied by changes in the expression levels of proteins in the eNOS/NO signaling axis; this mechanism was discovered to serve a key role in Hcy-induced endothelial dysfunction $(19,20)$. NO is widely accepted to be a potent endothelial relaxing factor, which is mainly produced by the eNOS-induced catalysis of L-arginine. NO produced by the endothelial cells diffuses to smooth muscle cells of the blood vessel and is activated by guanylate cyclization (21). Enzymes mediate the relaxation effect of the vascular smooth muscle, thereby regulating the vasodilation function (22). The eNOS/NO signaling axis is known to help maintain vascular homeostasis and has therefore attracted significant interest in the field of vascular pharmacology $(23,24)$. Accumulating evidence 
A

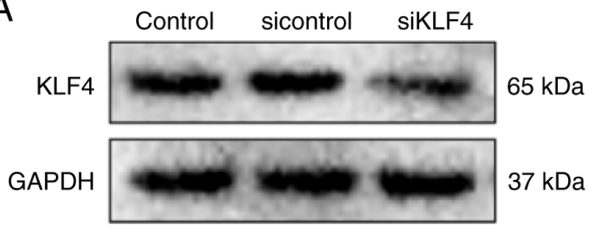

B
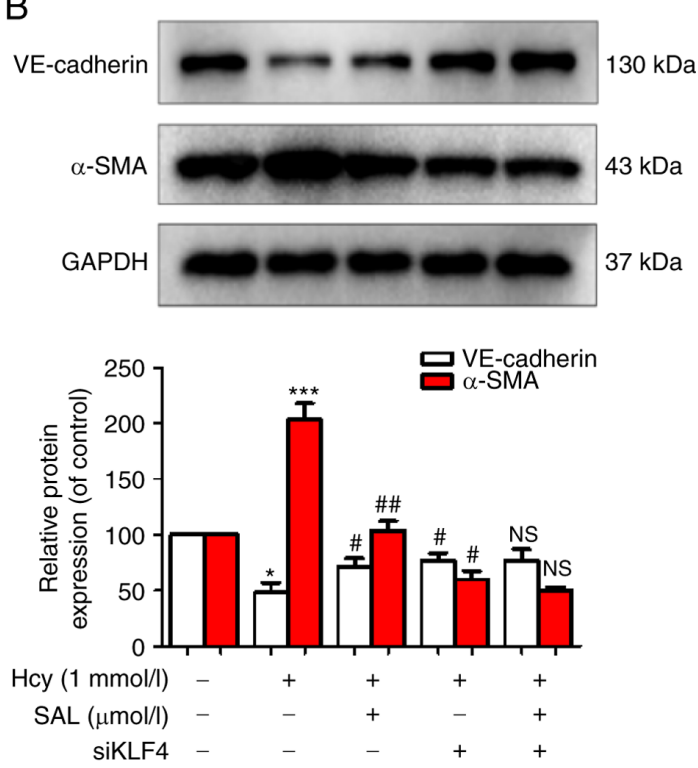

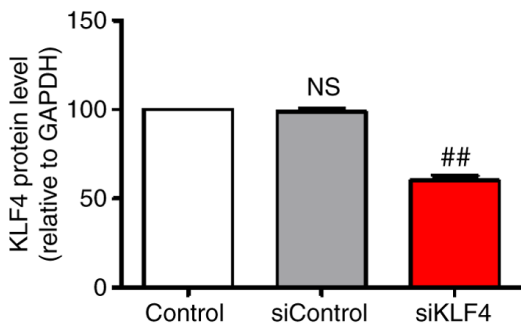

C
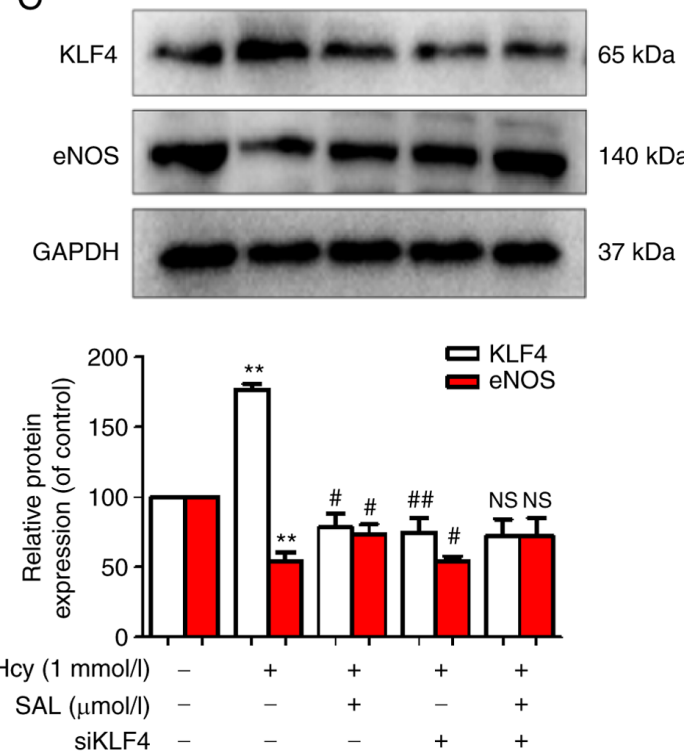

Figure 5. Effects of SAL and siKLF4 on the expression levels of Hcy-induced endothelial-mesenchymal transition-related markers, KLF4 and eNOS. (A) KLF4, (B) VE-cadherin and $\alpha$-SMA and (C) KLF4 and eNOS expression levels were analyzed using western blotting. Data are presented as the mean \pm SD of three independent experiments. " $\mathrm{P}<0.05,{ }^{* *} \mathrm{P}<0.01,{ }^{* * * *} \mathrm{P}<0.001$ vs. control; ${ }^{*} \mathrm{P}<0.05,{ }^{\# \#} \mathrm{P}<0.01$ vs. Hcy. Hcy, homocysteine; SAL, salidroside; KLF4, Kruppel-like factor 4; $\alpha$-SMA, $\alpha$-smooth muscle actin; eNOS, endothelial nitric oxide synthase; si, small interfering RNA; NS, not significant.

has suggested that eNOS/NO signaling axis was involved in vessel relaxation, vascular smooth muscle cell proliferation and ischemia/reperfusion injury (25). Moreover, our previous study revealed that KLF4 knockdown in endothelial cells could inhibit TGF- $\beta$-induced cell proliferation, migration and phenotypic changes, and effectively inhibited EndMT, suggesting that KLF4 may serve a role in EndMT (26). However, to the best of our knowledge, few studies have investigated the regulatory effect of the KLF4/eNOS signaling pathway in vascular damage-associated disorders. Our previous study reported that SAL upregulated the expression levels of the antioxidant signaling factor, Nrf2, which improved the Hcy-induced oxidative stress damage of endothelial cells, thereby suggesting its potential vascular pharmacological activity (13).

Based on the aforementioned findings, it was hypothesized that the KLF4/eNOS signaling pathway may serve a role in EndMT. Therefore, the present study aimed to investigate the effect and underlying molecular mechanisms of SAL in Hcy-induced EndMT. The current results revealed that Hcy treatment for $48 \mathrm{~h}$ significantly downregulated the expression levels of the endothelial specific marker, VE-cadherin, upregulated the expression levels of the mesenchymal cell marker, $\alpha$-SMA, and increased cell migration, indicating that Hcy may induce EndMT. Pretreatment with SAL inhibited these changes in the cell phenotypic markers and reduced cell migration, suggesting that SAL could effectively inhibit EndMT, and that its effect was associated with the upregulation of the eNOS/NO signaling axis and downregulation of KLF4 expression. The findings of the immunofluorescence assay demonstrated that Hcy induced the translocation of KLF4 from the cytoplasm to the nucleus, while the pretreatment with SAL reversed these effects, indicating that SAL intervention in EndMT may be association with the downregulation of the expression level of the TF KLF4. To further investigate whether KLF4 affected the eNOS/NO signaling axis, siRNA interference technology was used to knockdown KLF4 expression in HUVECs. The present findings showed that KLF4-knockdown increased the levels of cellular eNOS/NO signaling, suggesting that KLF4 may regulate the upstream molecular signaling of eNOS and participate in the regulation of vasodilation. However, no significant differences were observed between the SAL, siKLF4 and SAL + siKLF4 co-administration groups in the expression levels of the cell phenotypic markers, VE-cadherin and $\alpha$-SMA, indicating that KLF4 may represent a key molecular target for SAL to inhibit EndMT.

In conclusion, to the best of our knowledge, the results of the present study demonstrated, for the first time, that SAL may inhibit the effect of Hcy-induced EndMT by regulating the KLF4/eNOS signaling pathway; however, its precise underlying mechanism remains to be determined.

\section{Acknowledgements}

Not applicable. 


\section{Funding}

This work was funded by Scientific Research Project of Hunan Provincial Department of Education (grant no. 19B043), the Yan'an University Science Foundation Project (grant no. YDQ2020-06) and the Hunan Provincial Administration of Traditional Chinese Medicine Research Project Fund (grant no. 202083).

\section{Availability of data and materials}

The datasets used and/or analyzed during the current study are available from the corresponding author on reasonable request.

\section{Authors' contributions}

YH and XH designed and undertook experiments, and analyzed, interpreted and presented results for group discussions. YH, XH and JT performed some of the experiments and confirm the authenticity of all the raw data. XL provided methods, and was involved in the interpretation of results for the manuscript. XW made substantial contributions to the conception and design of the study. All authors have read and approved the final manuscript.

\section{Ethics approval and consent to participate}

Not applicable.

\section{Patient consent for publication}

Not applicable.

\section{Competing interests}

The authors declare that they have no competing interests.

\section{References}

1. Xu X, Friehs I, Hu TZ, Melnychenko I, Tampe B, Alnour F, Iascone M, Kalluri R, Zeisberg M, Del Nido PJ and Zeisberg EM: Endocardial fibroelastosis is caused by aberrant endothelial to mesenchymal transition. Circ Res 116: 857-866, 2015.

2. Bischoff J: Endothelial-to-mesenchymal transition. Circ Res 124: 1163-1165, 2019.

3. Qin W, Zhang L, Li Z, Xiao D, Zhang Y, Zhang H, Mokembo JN, Monayo SM, Jha NK, Kopylov P, et al: Endothelial to mesenchymal transition contributes to nicotine-induced atherosclerosis. Theranostics 10: 5276-5289, 2020.

4. Wadhera RK, Steen DL, Khan I, Giugliano RP and Foody JM: A review of low-density lipoprotein cholesterol, treatment strategies, and its impact on cardiovascular disease morbidity and mortality. J Clin Lipid 10: 472-489, 2016.

5. Guo Y, Li P, Bledsoe G, Yang ZR, Chao L and Chao J: Kallistatin inhibits TGF- $\beta$-induced endothelial-mesenchymal transition by differential regulation of microRNA-21 and eNOS expression. Exp Cell Res 337: 103-110, 2015.

6. Yoshida T, Yamashita M and Hayashi M: Kruppel-like factor 4 contributes to high phosphate-induced phenotypic switching of vascular smooth muscle cells into osteogenic cells. J Biol Chem 287: 25706-25714, 2012

7. Sun Q, Gong L, Qi R, Qing W, Zou M, Ke Q, Zhang L, Tang X, Nie Q, Yang Y, et al: Oxidative stress-induced KLF4 activates inflammatory response through IL17RA and its downstream targets in retinal pigment epithelial cells. Free Rad Biol Med 147: 271-281, 2020 .
8. Zhong Z, Han J,Zhang J, Xiao Q, Hu J and Chen L: Pharmacological activities, mechanisms of action, and safety of salidroside in the central nervous system. Drug Des Devel Ther 12: 1479-1489, 2018.

9. Zheng L, Su J, Zhang Z, Jiang L, Wei J, Xu X and Lv S: Salidroside regulates inflammatory pathway of alveolar macrophages by influencing the secretion of miRNA-146a exosomes by lung epithelial cells. Sci Rep 10: 20750, 2020.

10. Lai W, Xie X, Zhang X, Wang Y, Chu K, Brown J, Chen L and Hong G: Inhibition of complement drives increase in early growth response proteins and neuroprotection mediated by salidroside after cerebral ischemia. Inflammation 41: 449-463, 2018

11. Xing SS, Yang J, Li WJ, Li J, Chen L, Yang YT, Lei X, Li J, Wang K and Liu X: Salidroside decreases atherosclerosis plaque formation via inhibiting endothelial cell pyroptosis. Inflammation 43: 433-440, 2020.

12. Zhu L, Jia F, Wei J, Yu Y, Yu T, Wang Y, Sun J and Luo G: Salidroside protects against homocysteine-induced injury in human umbilical vein endothelial cells via the regulation of endoplasmic reticulum stress. Cardiovasc Ther 35: 33-39, 2017.

13. Zhang Yanyan, He Li, Huang Mei, Jiang Feng, Fu Lingyun and Li Jiachuan: Salidroside inhibits homocysteine induced oxidative stress in human umbilical vein endothelial cells. Journal of Southwest Minzu University (Natural Science Edition). 2020: 46 (04): 349-353. (In Chinese)

14. Xing SS, Li J, Chen L, Yang YF, He PL, Li J and Yang J: Salidroside attenuates endothelial cellular senescence via decreasing the expression of inflammatory cytokines and increasing the expression of SIRT3. Mech Ageing Dev 175: 1-6, 2018.

15. Livak KJ and Schmittgen TD: Analysis of relative gene expression data using real-time quantitative PCR and the 2(-Delta Delta C(T)) method. Methods 25: 402-408, 2001.

16. Song S, Zhang R, Cao W, Fang G, Yu Y, Wan Y, Wang C, Li Y and Wang Q: Foxm 1 is a critical driver of TGF- $\beta$-induced EndMT in endothelial cells through $\mathrm{Smad} 2 / 3$ and binds to the snail promoter. J Cell Physiol 234: 9052-9064, 2019.

17. van Putten S, Shafieyan Y and Hinz B: Mechanical control of cardiac myofibroblasts. J Mol Cell Cardiol 93: 133-142, 2016.

18. Gimbrone MA Jr and García-Cardeña G: Endothelial cell dysfunction and the pathobiology of atherosclerosis. Circ Res 19: 620-636, 2016.

19. Song CL, Liu B, Shi YF, Liu N, Yan YY,Zhang JC, Xue X, Wang JP, Zhao Z, Liu JG, et al: MicroRNA-130a alleviates human coronary artery endothelial cell injury and inflammatory responses by targeting PTEN via activating PI3K/Akt/eNOS signaling pathway. Oncotarget 7: 71922-71936, 2016.

20. Wang XJ, Tian DC, Wang FW, Zhang MH, Fan CD, Chen W, Wang MH, Fu XY and Ma JK: Astaxanthin inhibits homocysteine-induced endothelial cell dysfunction via the regulation of the reactive oxygen species-dependent VEGF-VEGFR2-FAK signaling pathway. Mol Med Rep 19: 4753-4760, 2019.

21. Rajendran S, Shen X, Glawe J, Gopi K, Kolluru GK and Kevil CG: Nitric oxide and hydrogen sulfide regulation of ischemic vascular growth and remodeling. Compr Physiol 12: 1213-1247, 2019.

22. Tan X, Feng L, Huang X, Yang Y, Yang C and Gao Y: Histone deacetylase inhibitors promote eNOS expression in vascular smooth muscle cells and suppress hypoxia-induced cell growth. J Cell Mol Med 21: 2022-2035, 2017.

23. Liu S, Sun Z, Chu P, Li H, Ahsan A, Zhou Z, Zhang Z, Sun B, Wu J, Xi Y, et al: EGCG protects against homocysteine-induced human umbilical vein endothelial cells apoptosis by modulating mitochondrial-dependent apoptotic signaling and PI3K/Akt/eNOS signaling pathways. Apoptosis 22: 672-680, 2017.

24. Arab HH, Salama SA and Maghrabi IA: Camel milk attenuates methotrexate-induced kidney injury via activation of PI3K/Akt/eNOS signaling and intervention with oxidative aberrations. Food Funct 9: 2661-2672, 2018.

25. Li J, Xiang X, Xu H and Shi Y: Cilostazol promotes angiogenesis and increases cell proliferation after myocardial ischemia-reperfusion injury through a cAMP-dependent mechanism. Cardiovasc Eng Technol 10: 638-647, 2019.

26. Zhang Y, Li C, Huang Y, Zhao S, Xu Y, Chen Y, Jiang F, Tao L and Shen X: EOFAZ inhibits endothelial-to-mesenchymal transition through downregulation of KLF4. Int J Mol Med 46: 300-310, 2020. International (CC BY-NC-ND 4.0) License. 